\title{
A GEOMETRIC METHOD FOR AUTOMATIC EXTRACTION \\ OF SULCAL FUNDI
}

$$
\text { By }
$$

C.-Y. Kao

M. Hofer

G. Sapiro

J. Stern

and

D. Rottenberg

IMA Preprint Series \# 2078

( November 2005)

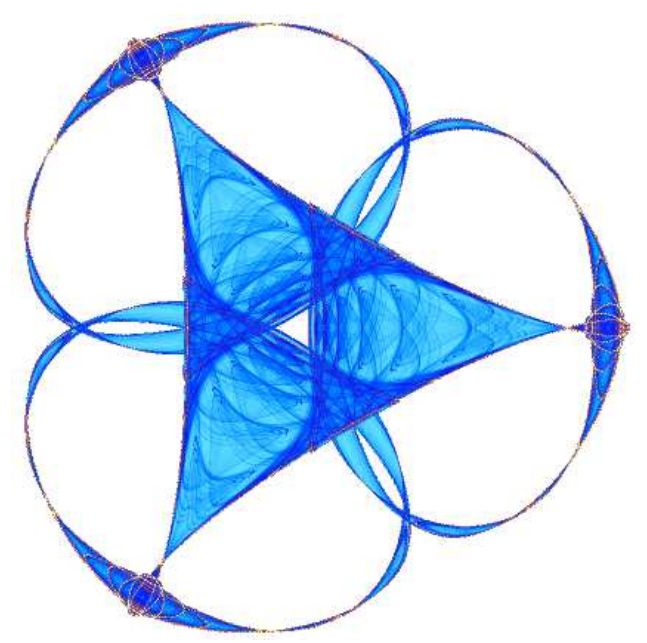

INSTITUTE FOR MATHEMATICS AND ITS APPLICATIONS

UNIVERSITY OF MINNESOTA 400 Lind Hall

207 Church Street S.E.

Minneapolis, Minnesota 55455-0436

Phone: 612/624-6066 Fax: 612/626-7370

URL: http://www.ima.umn.edu 


\title{
A GEOMETRIC METHOD FOR AUTOMATIC EXTRACTION OF SULCAL FUNDI
}

\author{
C.-Y. Kao ${ }^{1,4}$, M. Hofer ${ }^{6}$, G. Sapiro ${ }^{3}$, J. Stern ${ }^{1}$, and D.A. Rottenberg ${ }^{1,2,5}$ \\ ${ }^{1}$ Department of Neurology, University of Minnesota, Minneapolis, MN, USA \\ ${ }^{2}$ Department of Radiology, University of Minnesota, Minneapolis, MN, USA \\ ${ }^{3}$ Department of Electrical and Computer Engineering, University of Minnesota, Minneapolis, MN, USA \\ ${ }^{4}$ Institute for Mathematics and its Applications, University of Minnesota, Minneapolis, MN, USA \\ ${ }^{5}$ Minneapolis VA Medical Center, Minneapolis, MN, USA \\ ${ }^{6} \mathrm{TU}$ Vienna, Geometric Modeling \& Industrial Geometry, Vienna, Austria
}

\begin{abstract}
Sulcal fundi are 3D curves that lie in the depths of the cerebral cortex and are often used as landmarks for downstream computations in brain imaging. We present a sequence of geometric algorithms which automatically extract the sulcal fundi from magnetic resonance images and represent them as smooth polylines lying on the cortical surface. First we compute a geometric depth measure for each point on the cortical surface, and based on this information we extract sulcal regions by checking the connectivity above a depth threshold. We then extract the endpoints of each fundus and delineate the fundus by thinning each connected region keeping the endpoints fixed. The curves thus defined are smoothed using weighted splines on the gray-matter surface to yield highquality representations of the sulcal fundi
\end{abstract}

\section{INTRODUCTION}

When viewed from the outside a human brain appears as a volume with a highly wrinkled surface having numerous long crevices. The term sulci (plural of sulcus) is associated with these crevice regions and the term gyri (plural of gyrus) designates the regions between the sulci. In the computational neuroanatomical literature, 'sulcus' is used to describe the area of the pial surface within the sulcal depression and/or the volume of CSF contained therein. Accordingly, sulci have been represented as connected regions of the sulcal surface and as connected voxels lying within the sulcal depression. Informally, the fundus of a sulcus is the curve of maximal average "depth" that spans the length of the sulcus. The concepts of sulcal depth and fundus can be made precise in different ways; we introduce novel methods of defining sulcal depth and sulcal fundi below.

The importance of curvilinear representations of sulcal fundi lies in their use as landmarks for creating deformation

This work was partly supported by the NIH Human Brain Project P20 Grant EB02013, by the Austrian Science Fund under grant S9206, NSF, and NIH-Novel PDEs for Cortical Mapping and Analysis in Disease. fields for warping the cortical surfaces of different brains onto each other. The surface-to-surface warping approach has been used for longitudinal and cross-sectional studies of brain structure and function, cortical thickness, and gray-matter density [4]. The sulci and gyri form the highly convoluted cortex of the human brain. They serve as anatomical landmarks, and "segment" the cortex into functionally distinct regions. Although experts agree on the nomenclature for the major sulci, e.g., the central sulcus and Sylvian fissure, secondary and tertiary sulcal patterns vary greatly from individual to individual, and the nomenclature used by different anatomists is inconsistent. Sulcal endpoints and branchings are rarely defined, even for the major sulci.

Methods for extracting the cortical surface from MRI brain volumes have facilitated studies of intersubject gyral and sulcal variability. Traditionally cortical sulci and sulcal fundi have been manually defined by labeling voxels in an MRI brain volume using a GUI which displays only three orthogonal 2D brain slices. This process is extremely tedious and time consuming and, not surprisingly, prone to human error. Thompson et al. [4] manually drew 38 sulcal curves on MRI brain volumes acquired from a large number of normal subjects and patients with Alzheimer's disease and schizophrenia in order to identify characteristic patterns of brain structure and function. Given the large number of high-resolution MRI datasets currently available for analysis, automatic and objective extraction and labeling of cortical sulci has become a necessity.

Our primary motivation for the present work is the need for curves that accurately represent the sulcal fundi and can be used as input to brain-surface warping algorithms [16]. Additionally, we believe that automating sulcal extraction can improve the quality and reproducibility of the process as well as yielding considerable time savings. 

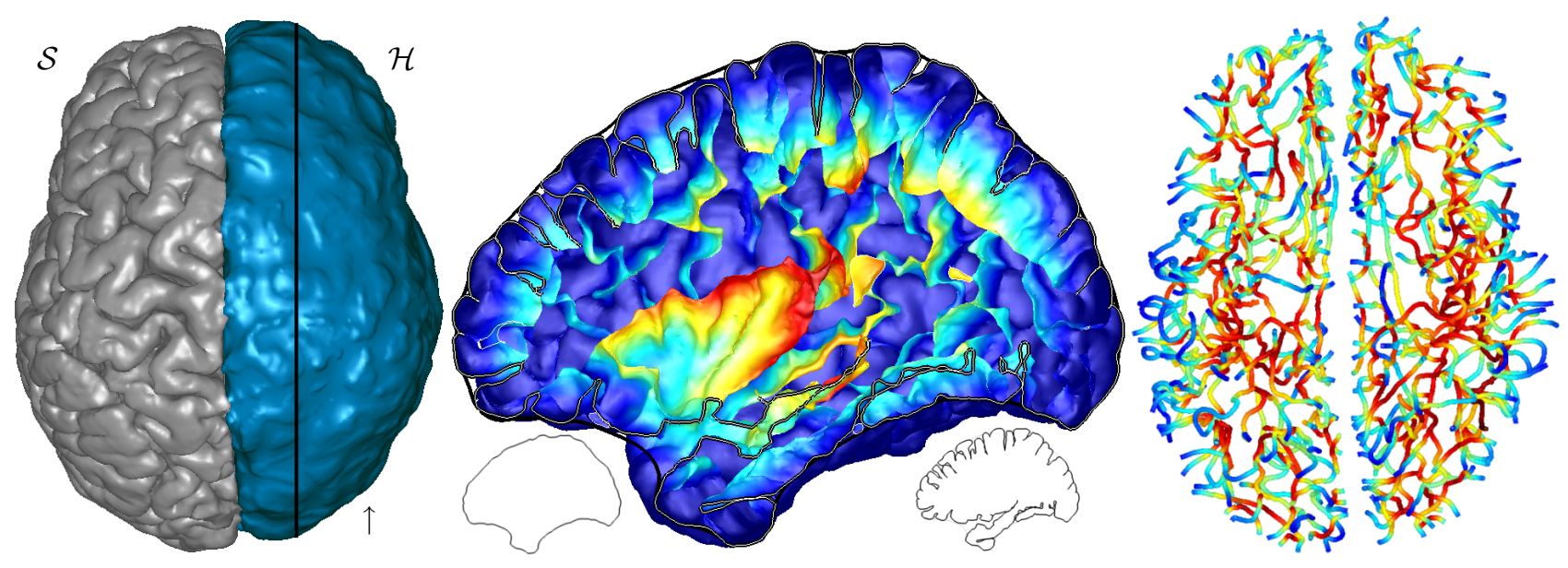

Fig. 1. Left. Pial surface $\mathcal{S}$ of the left hemisphere and outer hull $\mathcal{H}$ for the right hemisphere. The black line indicates the position of the cutting plane for the (middle) image. Middle. Side View of part of the right hemisphere (marked with an arrow) in the left-hand image. The two thumbnails illustrate the boundary curve of the hull and the pial surface, respectively. Right. Fully automatic extracted sulcal fundi represented as thick 3D curves. Colors in the middle and right-hand images indicate computed geodesic depth, from "shallow" (blue) to "deep" (red).

\subsection{Previous Work}

Previous work on automatically extracting curvilinear representations of sulcal fundi can be roughly divided into two approaches: those based on curvature and those based on distance functions. Curvature-based approaches define sulcal fundi as curves lying within areas of the extremal mean or principal surface curvature, whereas distance-based approached define them as curves whose distance to a hull bounding the cortical surface is locally maximal. Curvature-based approaches are often semi-automatic: the two end points of a sulcus are manually defined, and a curve connecting these points is then computed using (for example) dynamic programming [8], weighted geodesics computed by fast marching methods on triangular meshes [1], or fast marching methods on implicit surfaces [11]. Subvoxel tracking in volumetric data in the direction of the principal curvature has also been proposed [12].

Distance-based approaches often compute medial sulcal surfaces ("sulcal ribbons") from volumetric data and define the fundi as the inferior margins of these surfaces $[3,10]$ or as the projection of these margins onto a triangle mesh representing the cortical surface [2]. Previous work that combines curvature- and distance-based computations are semiautomatic algorithms that compute fundal curves using a modified fast-marching algorithm on triangular meshes [14] or on a flat map of the cortical surface [15].

\section{METHODS}

A fully automated method for the extraction of sulcal fundi from MRI brain images combines an automated method for extracting a 3D triangular mesh representation of the brain's cortical surface with an automated method for defining fundal curves that lie on the mesh surface. In this paper we do not introduce a new approach to cortical surface extraction; rather we describe a method for definining sulcal depth and sulcal fundi given a mesh representation of the gray-matter (GM) surface as an algorithmic input. Han et al. [5] provides an overview and discussion of methods that have been proposed to extract the cortical surface in implicit, parametric, or mesh representations. Additional publicly available surface extraction software methods include FreeSurfer and SurfRelax. ${ }^{1}$

\subsection{Segmentation and Surface Extraction}

The T1-weighted MRI brain volume used in this study $(1 \mathrm{~mm}$ isotropic voxels) was acquired at the Montreal Neurologic Institute and provided by Dr. Alan C. Evans. A topologicallycorrect triangular mesh, M, representing the pial (GM-CSF) surface of the cerebral cortex was extracted by FreeSurfer after skull stripping using BET ${ }^{2}$ Our approach to the definition of sulcal depth is based on a level set technique. In order to apply it the parametric and mesh representations are transformed into implicit form by computing the signed distance function to the surface on a grid using, for example, a fast sweeping algorithm [7]. In implicit form the pial surface is the zero level set $\Phi=0$ of an implicit function $\Phi$.

\footnotetext{
${ }^{1}$ FreeSurfer, see http://surfer.nmr.mgh.harvard.edu/, SurfRelax, see http://www.cns.nyu.edu/ jonas/software.html

${ }^{2} B E T$, see http://www.fmrib.ox.ac.uk/fsl/bet/.
} 


\subsection{Outer Hull Surface Extraction}

An outer hull surface $\mathcal{H}$ which warps the pial surface $\mathcal{S}$ is computed using a morphological closing operation applied to the level set function. The resulting outer hull is shown in Figure 1 (left). For morphological closing we move the surface outward by a time parameter $T$ and then move the surface inward by the same amount of time. The governing equation is

$$
\left\{\begin{array}{ccc}
\Phi_{t}+V(t)|\nabla \Phi| & = & 0 \\
\Phi(x, 0) & = & \Phi
\end{array}\right.
$$

where

$$
V(t)=\left\{\begin{array}{ccc}
1 & \text { for } & t \leq T \\
-1 & \text { for } & T<t \leq 2 T
\end{array}\right\}
$$

In our algorithm we choose $T=10$ ( $\mathrm{mm} /$ unit time). This is related to the width of sulcal regions. We want to choose the parameter $T$ to be large enough to close the sulcal regions and small enough to keep the overall shape of the brain.

\subsection{Geodesic Depth Computation}

After we obtain the outer hull surface we calculate the geodesic depth (distance) for any given point on the pial surface to the outer hull surface, see Figure 1 (middle) for the result. The particular geodesics that we want correspond to the shortest paths from each pial surface location to the outer hull which do not cross the surface. This can be efficiently computed by applying the fast sweeping method [7] to the restricted (CSF) region between the outer hull and the pial surface. The calculation is performed on a rectangular grid. Trilinear interpolation is used to propagate the depth information onto the triangular-mesh surface.

Our approach is different from that of previous work of [10] and [13] which either consider the Euclidean distance to the outer hull or the geodesic distance on the triangular mesh. Figure 2 illustrates why we prefer the geodesic distance within the restricted region. In [10], point $C$ and point $D$ are approximately the same Euclidean distance from the curve $h$, and in [13], point A and point B are approximately the same geodesic distance to the hull along the curve $s$. In our approach the order of the depth is $d(C)>d(B)>d(A) \cong$ $d(D)$ which is more anatomically correct. The geodesic calculation is done in $3 \mathrm{D}$.

\subsection{Sulcal Fundus Extraction}

The algorithmic steps described above result in the association of a sulcal depth estimate with each mesh triangle. Next, we use a depth threshold $D$ to define the sulcal regions of $S$ as those with a depth $d>D$, see Figure 2. In the literature $D$ is usually considered to be $2-3 \mathrm{~mm}$. Here we use $D=2.5 \mathrm{~mm}$. Within these sulcal regions we find the connected components $C_{i}$ by a connected components labeling algorithm, and for each component $C_{i}$ we compute the strip
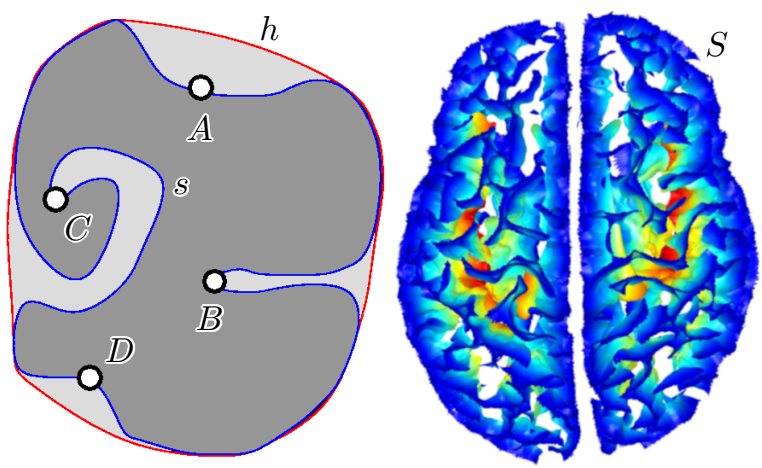

Fig. 2. Left. A 2D illustration motivating the depth computation (see text). Right. Remaining pial surface after removal of those segments with a geodesic "depth" less than $2.5 \mathrm{~mm}$.

$B_{i}$ of boundary triangles. The next stage of our algorithm identifies a small subset of each $B_{i}$ which constitutes the endpoints of the sulcus (a non-branching sulcus has exactly two endpoints; a branching sulcus is illustrated in Figure 3. The algorithm for identifying endpoints is based on the following concept: for each p in a given $B_{i}$, we associate a principal component direction with the set of points $N_{p}$ in a local neighborhood around $\mathrm{p}$, and we identify as endpoints those points $\mathrm{p}$ which are extremal according to the principal component direction in their local neighborhood $N_{p}$. We use a moving least squares (MLS) algorithm [9] to the compute the local principal component directions. Next we run a surface thinning algorithm that can be summarized as follows. Take those triangles of $B_{i}$ that correspond to the computed endpoints $p_{j}$ and add them to an initial skeleton list $S_{i}$. Then repeat the following two steps until all triangles of the component $C_{i}$ have been processed:

1. Find the triangle $\Delta$ of $B_{i}$ with the least depth.

2. If $\Delta$ is not connected to any interior triangles of $C_{i}$, then we add it to the skeleton list $S_{i}$. If $\Delta$ is connected to interior triangles of $C_{i}$, then remove it from $B_{i}$, and add its neighbors to $B_{i}$.

The result of the thinning algorithm is the skeleton $S_{i}$ of each connected component, which is made up of connected strips of triangles. We then use a minimum spanning tree algorithm to construct the tree structure of $S_{i}$. The longest non-branching path within the tree can be calculated by iteratively discarding the shortest branch leaving each vertex that has degree greater than two until only vertices of degree one and two remain.

\subsection{Sulcal Fundi Smoothing}

The extracted sulcal fundi are represented as polylines that are further smoothed by an algorithm that minimizes a counterpart to the cubic spline energy for curves on surfaces. We 


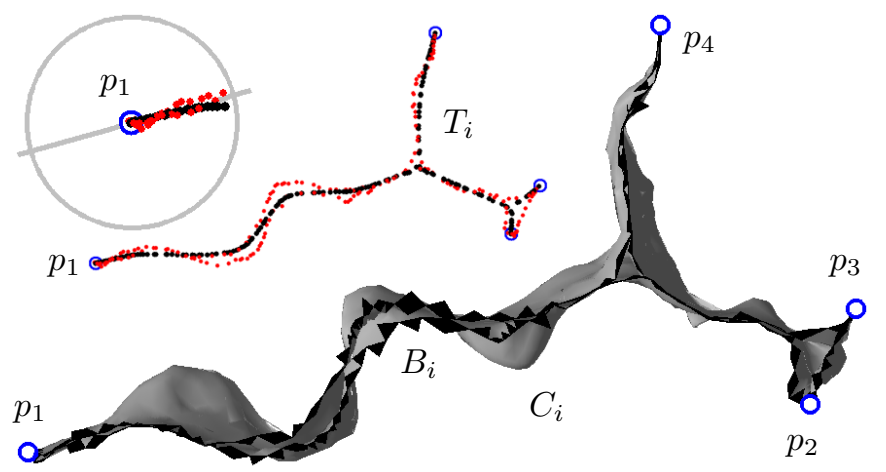

Fig. 3. Endpoints $p_{1}, \ldots, p_{4}$ of boundary $B_{i}$ of component $C_{i}$. The thin curve-like point set $T_{i}$ is computed using MLS. Endpoints $p_{j}$ are those points of $T_{i}$ that have in a ball neighborhood only neighbors in one direction.

extend the algorithm of [6] to weighted splines in manifolds $\mathbf{x}(u)$ that minimize the energy

$$
E_{2, w}(\mathbf{x}(u))=\int_{u_{1}}^{u_{n}} w(\mathbf{x}(u))\|\ddot{\mathbf{x}}(u)\|^{2} d u .
$$

The weight $w$ is a function depending on the mean curvature of the surface and the computed geodesic depth. If we want the curve to stay in the sulcal fundi then we have to choose a small weight $w$ for these regions. The basic form of the algorithm involves interleaving the steps of numerically minimizing the energy of a fully 3D parametric representation of a spline curve and projection of the curve to lie along the mesh surface.

\section{DISCUSSION}

Figure 1 (right) shows automatically extracted sulcal fundi in projection as thick $3 \mathrm{D}$ curves. The fundal curves automatically extracted by our algorithm (Figures 4 and 5) are similar to the "gold-standard" fundal outlines defined manually by an expert anatomist. The sympbols $\mathrm{X}, \mathrm{Y}$, and $\mathrm{Z}$ in Figure 4 indicate where the two results diverge. Aside from potential shortcomings in our definition of sulcal depth and fundal location there are several possible reasons for this divergence including errors in the underlying extracted mesh surface and errors in the manual labeling. All automatically extracted sulcal fundi are compared with 12 hand-labeled fundi (six in each hemisphere). Detailed views of the superior frontal sulcus reflect the accuracy of the automatically extracted fundi. (All figures of the paper are in color.)

\section{REFERENCES}

[1] A. Bartesaghi and G. Sapiro. A system for the generation of curves on 3D brain images. Human Brain Mapping, 14:1-15, 2001.

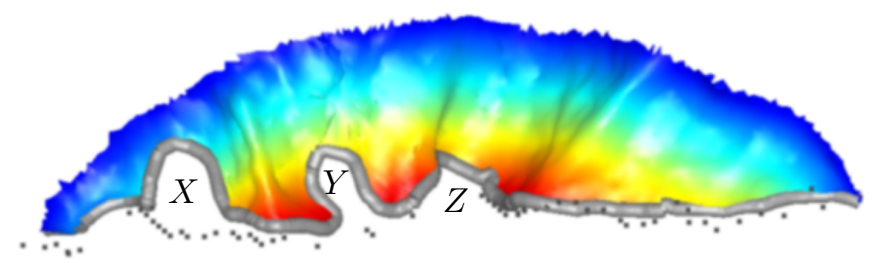

Fig. 4. Comparison of the "fundus" of the central sulcus extracted by our algorithm (thick gray curve) and the fundus delineated manually by an expert anatomist (black points). See text for details explaining $X, Y, Z$.

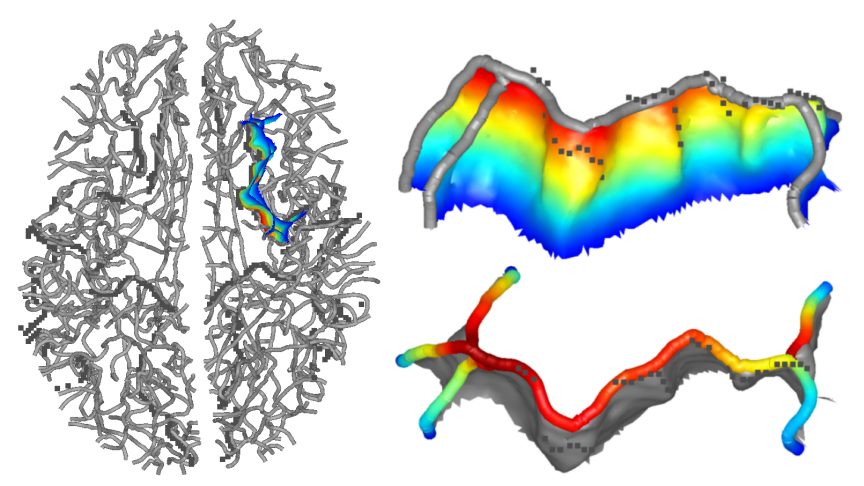

Fig. 5. Left. Comparison of all sulcal fundi extracted by our algorithm (thick gray curves) to six major fundi delineated manually by an expert anatomist (black points). Right. Two views of this comparison for the superior frontal sulcus. 
[2] A. Cachia et al. A primal sketch of the cortex mean curvature: a morphogenesis based appraoch to study the variability of the folding patterns. IEEE Trans. on Med. Imaging, 22(6):754-765, 2003.

[3] G. Le Goualher et al. Automated extraction and variability analysis of sulcal neuroanatomy. IEEE Trans. Med. Imaging, 18(3):206-217, 1999.

[4] P.M. Thompson et al. Mapping cortical change in Alzheimer's disease, brain development, and schizophrenia. NeuroImage, 23(S1):2-18, 2004.

[5] X. Han et al. CRUISE: cortical reconstruction using implicit surface evolution. NeuroImage, 23:997-1012, 2004.

[6] M. Hofer and H. Pottmann. Energy-minimizing splines in manifolds. Trans. on Graphics, 23(3):284-293, 2004.

[7] C.-Y. Kao, S. Osher, and Y.-H. Tsai. Fast sweeping methods for static Hamilton-Jacobi equations. SIAM Numerical Analysis, 42:2612-2632, 2005.

[8] N. Khaneja, M.I. Miller, and U. Grenander. Dynamic programming generation of curves on brain surfaces. IEEE PAMI, 20:1260-1265, 1998.

[9] D. Levin. The approximation power of moving leastsquares. Math. Comput., 224(67):1517-1531, 1998.

[10] G. Lohmann. Extracting line representations of sulcal and gyral patterns in MR images of the human brain. IEEE Trans. on Med. Imaging, 17(6):1040-1048, 1998.

[11] F. Mémoli, G. Sapiro, and P. Thompson. Implicit brain imaging. NeuroImage, 23(S1):179-188, 2004.

[12] C. Renault, M. Desvignes, and M. Revenu. 3D curves tracking and its application to cortical sulci detection. ICIP 2000, 2:491-494, 2000.

[13] M.E. Rettmann, X. Han, C. Xu, and J.L. Prince. Sulcal segmentation using watersheds on the cortical surface. NeuroImage, 15:329-344, 2002.

[14] X. Tao, X. Han, M.E. Rettmann, J.L. Prince, and C. Davatzikos. Statistical study on cortical sulci of human brains. In Proc. IPMI'01, pages 475-487, 2001.

[15] X. Tao, J.L. Prince, and C. Davatzikos. An automated method for finding curves of sulcal fundi on human cortical surfaces. In ISBI, pages 1271-1274, 2004.

[16] A.W. Toga. Brain Warping. Academic Press, New York, 1998. 